\title{
Building a Website Architecture based on Face Authentication for Developing Trust between Consumers in KSA
}

\author{
Mohamed S. Farag \\ Math Department, Computer Science \\ Al-Azhar University, Cairo, Egypt Egypt\& \\ Community College, Majmaah University, Saudi Arabia
}

\begin{abstract}
Millions of Internet users worldwide are engaged in buying and selling of various products using Consumers (C2C) online buy and sell websites, The aim of this study is to working on increasing the trust in e-commerce between Consumers in the Kingdom of Saudi Arabia(KSA), about studying the architecture of the most important websites (Taobao, eBay and Paipai,...), which use the sale and purchase between consumers, and extract the problems that correspond to consumers which using of these sites and avoid them. One of the most important reasons that lead to increased trust between consumers is the ability of a trusted third parties (TTP) to be sure from both parties buyer and seller, so in this study we will discuss a number of algorithms used in face recognition and propose a more efficient algorithm for face recognition and implement it, to be sure from consumers entering the website (increasing the website authentication security). Finally we proposed architecture for our website, which will be used in buying and selling in KSA, this architecture consists of three stages, first stage: the stage of registration for the first time and where the consumer fills out the application form. The second stage: The login stages the consumer entering Assurance Key (AK), and uploads a personal image using webcam to $\log$ on to the website. the third stage: if TTP verify from the data in the first stage, generates AK for the consumer and send it to him, and in the second case, if TTP verify from the login data (authenticity of data) then the consumer can access the operations on the website.
\end{abstract}

\section{General Terms}

Pattern Recognition, Trust in E-Commerce, Security.

\section{Keywords}

Assurance keys (AK's), consumer to consumer, ECommerce, Face Recognition, evaluating a model.

\section{INTRODUCTION}

At present, many $\mathrm{C} 2 \mathrm{C}$ websites such as Taobao, eBay and so on have built their own online credit evaluation systems. The fundamental functions of which can be described as follows: Both parties of a transaction evaluate the other party's behavior each other during a specified period of validity. Based on the feedback information, a participant's credit and behavior records are aggregated and conveyed in a specified way. As a tool of disseminating reputation, the online credit evaluation system facilitates building trust, encouraging trustworthy behavior, and deterring participation by those who are unskilled or dishonest. Thus they contribute to reducing the transaction risks, increasing the rate of successful transactions. Face recognition technology can be used in wide range of applications such as identity authentication, access control, and surveillance and security. Interests and research activities in face recognition have increased significantly over the past few years.

\section{LITERATURE REVIEW}

The literature review falls into three main parts: part one focus on models, which will discuss the trust that touched upon the relationship of consumers with each other, part two on websites, in this part analyzing the most common websites in $\mathrm{C} 2 \mathrm{C}$ e-commerce determining both the future and limitation and part three focus on studies of previous systems of face recognition (the systems of face recognition in the field of web authentication of $\mathrm{C} 2 \mathrm{C}$ ).

\subsection{Part_1: Models for C2C}

2.1.1 Trust in virtual communities and its effect on consumers' purchase intention [1]

This research analyzes what factors affect trust building among virtual community (VC) members and how this trust influences the trust in the $\mathrm{C} 2 \mathrm{C}$ website or vendor fig. 1, propose and empirically test a model of trust in VCs based on the trust formation mechanism fig. 2, Using data collected from Taobao Virtual Community.

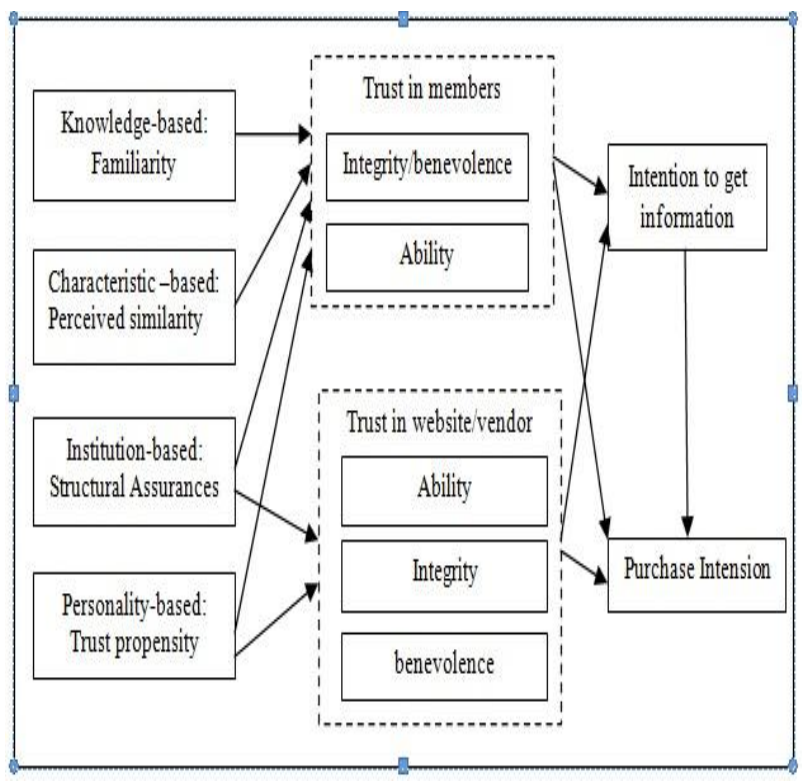

Fig 1: The research model 


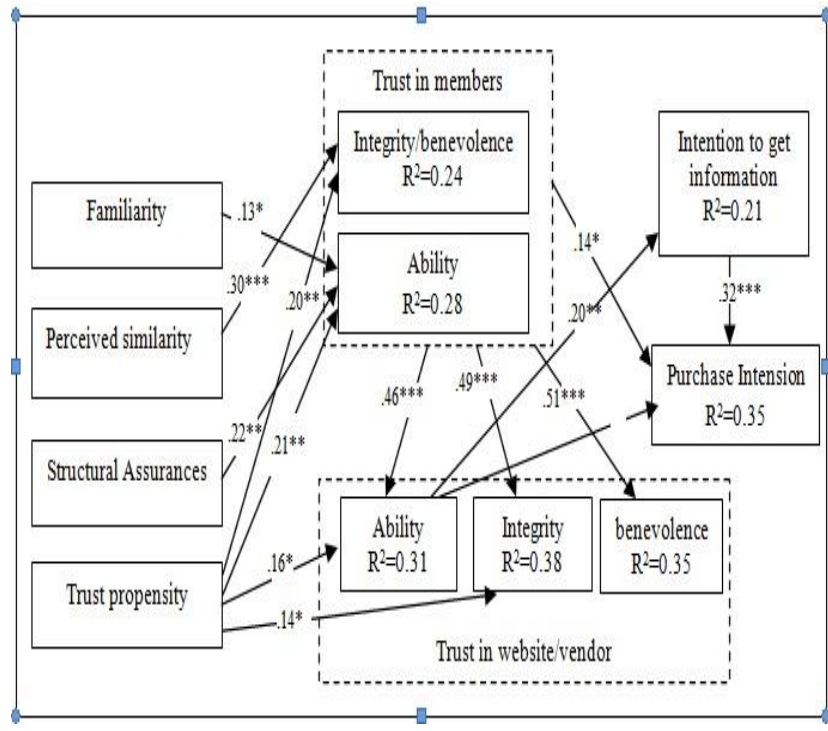

Fig 2: Standardized LISREL solution (model with only significant paths). Note $:{ }^{*} p<0.05 ;{ }^{* *}$ p $<0.01 ;{ }^{* * *}$ p $<0.001$

\subsubsection{Credit Evaluation Model for C2C [2-4]}

This paper first introduced existing credit evaluation model for $\mathrm{C} 2 \mathrm{C}$ e-commerce, and analyzed the major problems in it. Then an improved credit evaluation model for $\mathrm{C} 2 \mathrm{C}$ ecommerce is created, which contributed in solving the problems existed in models and established more practicable and effective credit evaluation systems for $\mathrm{C} 2 \mathrm{C}$ ecommerce. , see fig. 3 .

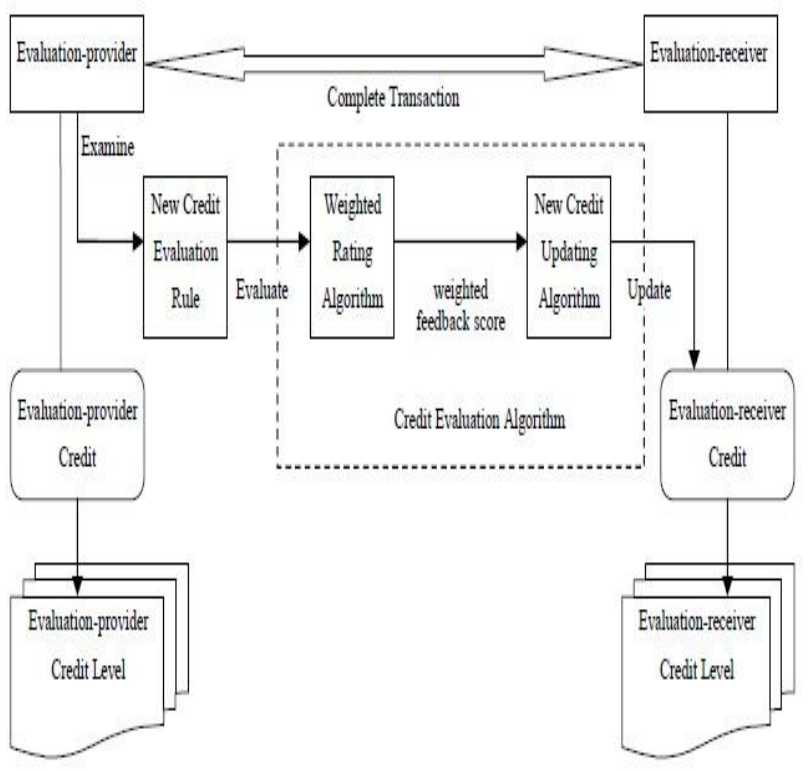

Fig 3: The improved credit evaluation model for $\mathrm{C2C}$

$$
\text { e-commerce }
$$

\subsubsection{PayPal Credit evaluation model[5]}

eBay uses a system for verification from the other party by PayPal, which in turn depends on the registered credit card to verify from the consumer. Several companies of credit cards generate their own unique numbers. The major companies of credit cards use the same method for generating their numbers.

\subsubsection{Dehua HE Model [6]}

Despite the increasing importance of E-Commerce, there is a major barrier, a lack of intent to purchase, so there is a need to strengthen consumers' intentions to buy online. This paper assumes that the reasons influencing the purchase intention are five:

i. Ease of use of the site.

ii. To look at the extent of use of the site.

iii. Seller specialized (high-efficiency).

iv. Recommendations of a third party.

v. Position of vendors towards customers.

\subsection{4 eBay Model [5],[7]}

eBay is one of the leading company in the field of ecommerce and created one of the most common trusted online commercial communities, whereby the transactional exchange between sellers and buyers is regulated by the evaluations and recommendations of each.

\subsection{Part_2: Websites for C2C}

Previous models have been applied in practice and spread in a recent E-Commerce sites between C2C. Some of these sites work on display and sale in a specialized field such as cars or real estate. There are sites that work on the presentation of several different products while other sites work on display services, e.g.

\subsubsection{Mudah Website [8]}

Malaysia is one of the advanced countries in the field of technology and so like many countries having a website (Mudah.my) for electronic commerce, which allows the purchases and sales for small and medium business and home, which is e-commerce between individuals, this website have more than 5.2 million visitors a month, and is one of the most visited websites in Malaysia. Which offer special services for professional advertisers, and has more than 84,000 interfaces of stores and helps small and medium enterprises and home based businesses to advertise their products and services on the internet. So Mudah.my is conceder the largest online marketplace in Malaysia.

\subsubsection{Haraj Website [9]}

This is a Saudi (KSA) website that is dedicated to cars, electronics and real estate. The website receives a commission of $1 \%$ from the seller after the transaction is completed.

\subsubsection{Autotrader Website [10]}

This site is in Britain; it is a specialized in buying and selling cars. AutoTrader.com has been extremely successful in applying SAS to manage and report voluminous business information since April 1999, and successfully rose to each new level.

\subsubsection{Witkey Website [1]}

E-commerce websites between $\mathrm{C} 2 \mathrm{C}$ are mostly focused on the buying and selling of products of various kinds in order to return the material, but in the last few years have appeared very different types of websites, said to be $\mathrm{C} 2 \mathrm{C}$ An example of this type of website is Witkey, working on the sale and purchase of products property such as designs logos, translation of articles and writing documents. Witkey is a 
typical website in China and has grown rapidly in the past few years, which led to interest in VC in this matter.

\subsection{5 eBay Website [12 -13]}

eBay is the world's online marketplace; a place for buyers and sellers to come together and trade almost anything. eBay, Inc. is one of the most successful Web based enterprise in existence. Its name is universally known and is synonymous with the auction model of Internet selling. eBay was pivotal in helping to facilitate buying and selling between individuals and businesses. The industry leader also created one of the first trusted online commercial communities, whereby the transactional exchange between sellers and buyers is regulated by the evaluations and recommendations of each.

\subsection{Part_3: Face Recognition}

Face recognition is the most commonly used method of authenticating a computer user is via username and password. Many companies have discovered that passwords can be guessed, stolen or forgotten. They can often be cracked using tools freely available on the internet. Multiple passwords per user is not feasible, as this is inconvenient for the user, difficult to remember, and time consuming for administrators. Face recognition can either act as an alternative to the password system, or add an extra layer of security to the existing system. The choices of the face recognition algorithms includes template-based approaches, such as SVM, PCA, LDA, ICA, FDA, Bayesian and feature-based-one such as the proprietary algorithm.

\subsubsection{PCA Algorithm [14-15]}

The goal of PCA is to reduce the dimensionality of the data while retaining as much as possible of the variation present in the dataset. The flowchart algorithm see fig. 4, the eigenfaces approach for face recognition involves the following initialization operations:

1. Acquire a set of training images.

2. Calculate the eigenfaces from the training set, keeping only the best $\mathrm{M}$ images with the highest eigenvalues. These M images define the "face space". As new faces are experienced, the eigenfaces can be updated.

3. Calculate the corresponding distribution in M-dimensional weight space for each known individual (training image), by projecting their face images onto the face space.

Calculating Eigenfaces: Let a face image $\Gamma(\mathrm{x}, \mathrm{y})$ be a twodimensional $\mathrm{N}$ by $\mathrm{N}$ array of intensity values. An image may also be considered as a vector of dimension $N^{2}$, Let the training set of face images be $\Gamma_{1}, \Gamma_{2}, \Gamma_{3}, \ldots, \Gamma_{M}$. The average face of the set if defined by $\Psi=\frac{1}{M} \sum_{n=1}^{M} \Gamma_{n}$. Each face differs from the average by the vector $\Phi_{n}=\Gamma_{n}-\Psi$. An example training set is shown in fig. 1a, with the average face $\Psi$ shown in fig. 1b. This set of very large vectors is then subject to principal component analysis, which seeks a set of $\mathrm{M}$ orthonormal vectors, $\mu_{n}$, which best describes the distribution of the data. The $\mathrm{k}^{\text {th }}$ vector, $\mu_{k}$ is chosen such that

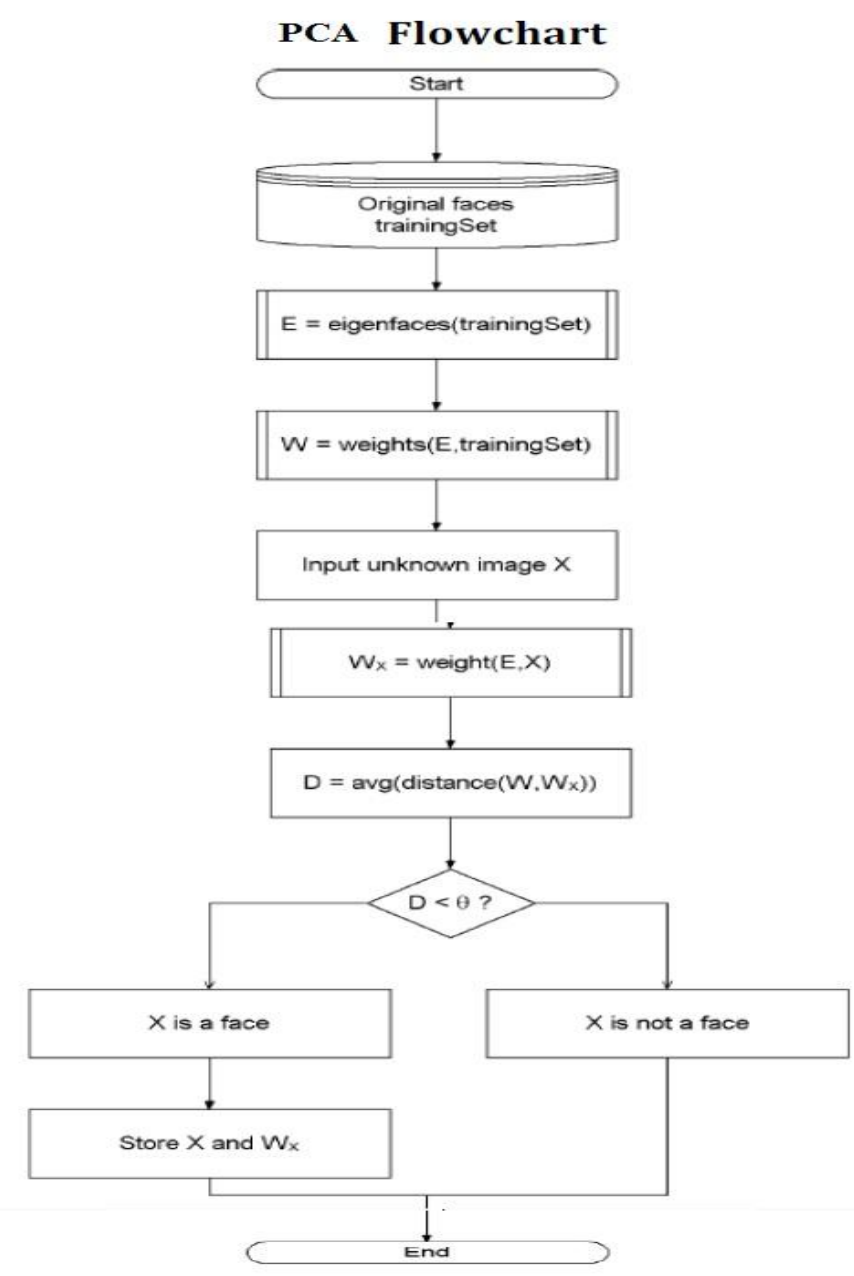

Fig 4: Flowchart of PCA algorithm

$\lambda_{k}=\frac{1}{M} \sum_{n=1}^{M}\left(\mu_{k}^{T} \Phi_{n}\right)^{2}$

is a maximum, subject to

$\mu_{l}^{T} \mu_{k}=\left\{\begin{array}{l}1, l=k \\ 0, \text { otherwise }\end{array}\right.$

The vectors $\mu_{k}$ and scalars $\lambda_{k}$ are the eigenvectors and eigenvalues, respectively, of the covariance matrix

$C=\frac{1}{M} \sum_{n=1}^{M} \Phi_{n} \Phi_{n}^{T}=A A^{T}$

where the matrix $A=\left[\Phi_{1} \Phi_{2} \ldots \Phi_{M}\right]$. The matrix C, however, is $N^{2}$ by $N^{2}$, and determining the $N^{2}$ eigenvectors and eigenvalues is an intractable task for typical image sizes. A computationally feasible method is needed to find these eigenvectors.

If the number of data points in the image space is less than the dimension of the space $\left(M<N^{2}\right)$, there will be only $M-1$, rather than $N^{2}$, meaningful eigenvectors (the remaining eigenvectors will have associated eigenvalues of 
zero). Fortunately, we can solve for the $N^{2}$ - dimensional eigenvectors in this case by first solving for the eigenvectors of and $\mathrm{M}$ by $\mathrm{M}$ matrix - e.g., solving a $16 \times 16$ matrix rather than a $16,384 \times 16,384$ matrix - and then taking appropriate linear combinations of the face images $\Phi_{n}$. Consider the eigenvectors $v_{n}$ of $A^{T} A$ such that

$$
A^{T} A v_{n}=\lambda_{n} v_{n}
$$

Pre-multiplying both sides by A, we have

$$
A A^{T} A v_{n}=\lambda_{n} A v_{n}
$$

from which we see that $A v_{n}$ are the eigenvectors of $C=A A^{T}$.

Following this analysis, we construct the $\mathrm{M}$ by $\mathrm{M}$ matrix $L=A^{T} A$, where $L_{m n}=\Phi_{m}^{T} \Phi_{n}$, and find the $\mathrm{M}$ eigenvectors $v_{n}$ of $\mathrm{L}$. These vectors determine linear combinations of the $\mathrm{M}$ training set face images to form the Eigen faces $\mu_{n}$ :

$$
\mu_{n}=\sum_{k=1}^{M} v_{n k} \Phi_{k}=A v_{n}, n=1, \ldots \ldots, M
$$

With this analysis the calculations are greatly reduced, from the order of the number of pixels in the images $\left(N^{2}\right)$ to the order of the number of images in the training set (M). In practice, the training set of face images will be relatively small $\left(M<N^{2}\right)$, and the calculations become quite manageable. The associated eigenvalues allow us to rank the eigenvectors according to their usefulness in characterizing the variation among the images.

\subsubsection{SVM [ 16$]$}

The main idea of the SVM algorithm is that given a set of points which belong to one of the two classes, it is needed an optimal way to separate the two classes by a hyper plane as seen in the below fig. 5. This is done by:

- maximizing the distance (from closest points) of either class to the separating hyper plane see fig. 5 .

- minimizing the risk of misclassifying the training samples and the unseen test samples.

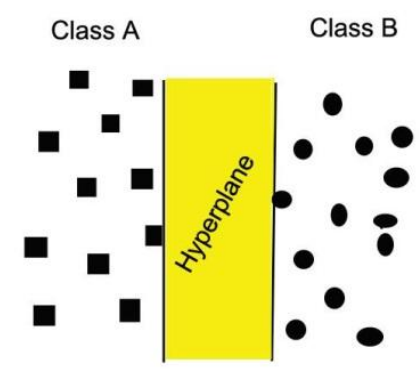

Fig 5: Optimal Separating Hyperplane

Depending on the way the given points are separated into the two available classes, the SVMs can be:

- Linear SVM see fig. 6.a

- Non-Linear SVM see fig. 6.b

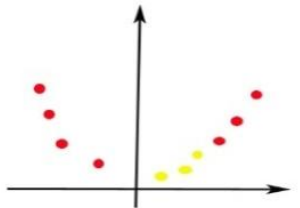

a) Linearly separable data b) Non Linearly separable data

Fig. 6: Linearly and non-linearly separable data Let $S$ be a set of points $x_{i} \in R^{d}$ with $i=1, \ldots m$. Each point $x_{i}$ belongs to either of two classes, with label $y_{i} \in\{-\mathbf{1}+\mathbf{1}\}$. The set $S$ is linear separable if there are $w \in R^{d}$ and $w_{0} \in R$ such that

$y_{i}\left(w \cdot x_{i}+w_{0}\right) \geq 1, \quad i=1, \ldots ., m \rightarrow 2.3 .2 .1$

The pair $\left(w, w_{0}\right)$ defines the hyper plane equation $w \cdot x+w_{0}=0$, named the separating hyper plane. The signed distance $d_{i}$ of a point $x_{i}$ to the separating hyper plane $\left(w, w_{0}\right)$ is given by:

$$
d_{i}=\frac{w \cdot x_{i}+w_{0}}{\|w\|}
$$

From (2.3.2.1) and (2.3.2.2) it follows that:

$$
y_{i} d_{i} \geq \frac{1}{\|v\|}
$$

therefore $\frac{1}{\|w\|}$ is the lower bound on the distance between points $x_{i}$ and the separating hyper plane $\left(w, w_{0}\right)$. Given a linearly separable set $S$, the optimal separating hyper plane is the separating hyper plane for which the distance to the closest (either positive or negative) points in $\mathrm{S}$ is maximum, therefore it maximizes $\frac{1}{\|w\|}$, see fig. 7 .

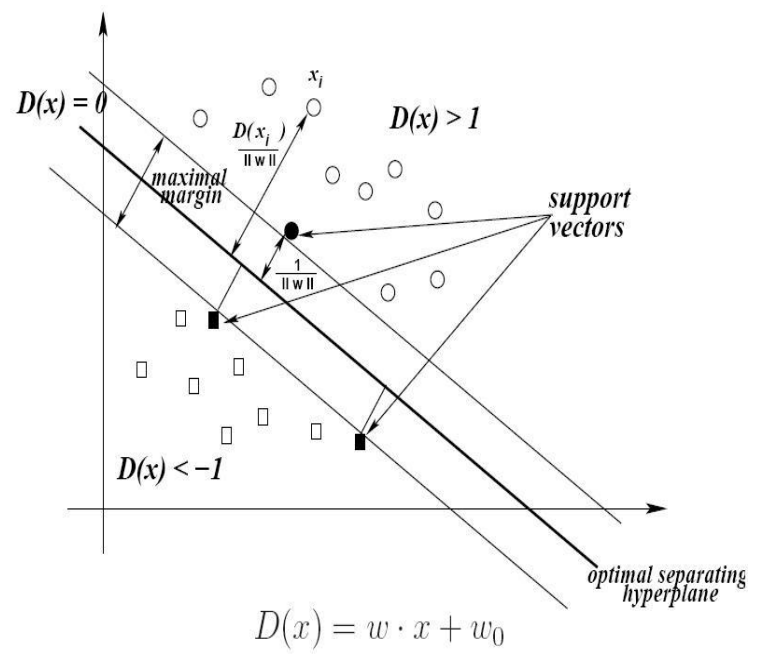

Fig 7: Optimal separating hyper plane 


\subsubsection{LDA and FDA [16-18]}

The goal of Linear Discriminant Analysis (LDA) is:

-Perform dimensionality reduction "while preserving as much of the class discriminatory information as possible".

-Seeks to find directions along which the classes are best separated.

-Takes into consideration the scatter within-classes, but also the scatter between-classes.

- For example in our case of face recognition, more capable of distinguishing image variation, due to identity from variation due to other sources such as illumination and expression.

Fisher Linear Discriminant (FLD) Analysis is an efficient discriminant model for face recognition. It aims to find a set of most discriminative linear projections by maximizing the ratio of the determinant of the between-class scatter matrix to that of the within-class scatter matrix:

$$
W_{o p t}=\arg \max _{w} \frac{\left\|W^{T} S_{B} W\right\|}{\left\|W^{T} S_{w} W\right\|}
$$

The within-class scatter matrix $S_{w}$ and between-class scatter matrix $S_{B}$ are respectively defined as

$$
\begin{array}{cc}
\sum_{i=1}^{c} \sum_{x \in X_{i}}\left(x-m_{i}\right)\left(x-m_{i}\right)^{T} & \rightarrow 2 \\
S_{B=\sum_{i=1}^{c} N_{i}\left(m_{i}-m\right)\left(m_{i}-m\right)^{T}} & \rightarrow 3
\end{array}
$$

Where $C$ is the number of classes in the training set, $N_{i}$ is the number of samples from class $i, m_{i}$ is the mean of all samples in class $i$, and $m$ is the mean of all the samples in the training set. From (2), we can see that, more than one sample is needed to calculate the $S_{W}$. So in the case of Single Sample Per Person (SSPP), $S_{B}$ degenerates to 0 , then any $W$ can maximize (1) to be infinite which means FLD fails to work.

\section{METHOD RESEARCH MODEL AND HYPOTHESES}

According to previous studies in literature review, we present our proposed website architecture see fig. 10, to develop trust in the E-Commerce between the $\mathrm{C} 2 \mathrm{C}$ and that in turn lead to the establishment of lasting relationships of trust between consumers and then work to expand the consumer base.

The idea of the website architecture based on several steps namely:

I- Consumers start up in the TTP and save their data in the database of TTP, and then gives AK, as well as to store the image in the database with AK.

II- Verification of the consumer (seller, buyer) before any electronic transaction, whether to view or purchase will be undertaken by online verification (OV) using $\mathrm{AK}$ and face recognition (FR).
III- After verifying the consumer by AK and FR and make sure it does not exist in the black list (BL), allows them to practice the process of electronic.

IV- Payment method depends on the account number registered in the TTP. Transfer is made directly to the seller's account whether from the buyer's account registered on TTP or any other method selected by the buyer. A copy of the payment receipt is sent to the TTP. V- A lasting relationship to build a trust community: If the purchase was made from the seller, a lasting trustbased relationship can be established between the buyer and the seller for future transactions.

This model to develop trust in the E-Commerce between the $\mathrm{C} 2 \mathrm{C}$ and that in turn lead to the establishment of lasting relationships of trust between consumers and then work to expand the consumer base. The TTP, inspection of goods before they are sent to the buyer, and if there be any fraud in the specification data is sent to the seller BL. In the event that the buyer the option to request payment upon receipt of payment of freight charges and the percentage of commission for TTP, and if the buyer did not adhere to the previous fees to pay the remaining amount, it sends its data to the BL.

\section{METHODOLOGY}

The methodology building a system based on two main parts: part one focuses on modify our proposed algorithm [19], for generating and validating AK to be suitable to be apply in KSA and proposed a multi-modal algorithm for face recognition, which will be used in web based authentication. The second part focuses on the proposed website architecture.

\subsection{Modifying algorithm [19], for generating $\mathrm{AK}$ and propose a multi modal algorithm for face recognition:}

\subsubsection{AKs Database}

The assurance key consists of four parts, the first part C, of 2-digit used for the city code see table 1 . We divided the country KSA into 20 regions using clustering (the largest cities), where every city and its nearest neighbors, the second part (AK-1) and the third part (AK-2) are random numbers each one of them contains 6-digits for each consumer and the fourth part (AK-V) of 4-digits used for validation the Assurance key deciding if it's in AK Database or not. Generating both parts AK1 and AK2 see fig. 8, without repetition and adjust all the generated random numbers to be 12-digits, as in the following algorithm:

1. Read the amount of random numbers that need to be generated(amount).

2. Read the minmum value of the range(MIN).

3. Read the maximum value of the range(MAX).

4. Define the range it must be at least equal the amount.range $=(\mathrm{MAX}-\mathrm{MIN})+1$; 
Table1: The range of people in largest cities of KSA.

\begin{tabular}{|c|c|c|c|c|c|}
\hline City Name & City code & Range of people & City Name & City code & Range of people \\
\hline$\underline{\text { Riyadh }}$ & 1 & $1-10,000,000$ & $\underline{\text { Tabuk }}$ & 11 & $1-2,000,000$ \\
\hline$\underline{\text { Jeddah }}$ & 2 & $1-10,000,000$ & $\underline{\text { Ha'il }}$ & 12 & $1-2,000,000$ \\
\hline$\underline{\text { Mecca }}$ & 3 & $1-5,000,000$ & $\underline{\text { Hafar Al- }}$ & 13 & $1-2,000,000$ \\
\hline$\underline{\text { Medina }}$ & 4 & $1-5,000,000$ & $\underline{\text { Jubail }}$ & 14 & $1-2,000,000$ \\
\hline$\underline{\text { Al-Ahsa }}$ & 5 & $1-3,000,000$ & $\underline{\text { Al-Kharj }}$ & 15 & $1-2,000,000$ \\
\hline$\underline{\text { Ta'if }}$ & 6 & $1-3,000,000$ & $\underline{\text { Qatif }}$ & 16 & $1-2,000,000$ \\
\hline$\underline{\text { Dammam }}$ & 7 & $1-3,000,000$ & $\underline{\text { Abha }}$ & 17 & $1-2,000,000$ \\
\hline$\underline{\text { Khamis }}$ & 8 & $1-3,000,000$ & $\underline{\text { Najran }}$ & 18 & $1-2,000,000$ \\
$\underline{\text { Mushait }}$ & 9 & $1-3,000,000$ & $\underline{\text { Yanbu }}$ & 19 & $1-2,000,000$ \\
\hline$\underline{\text { Buraidah }}$ & 9 & $1-3,000,000$ & $\underline{\text { Tabuk }}$ & 20 & $1-2,000,000$ \\
\hline$\underline{\text { Khob }}$ & 10 & & & & \\
\hline
\end{tabular}

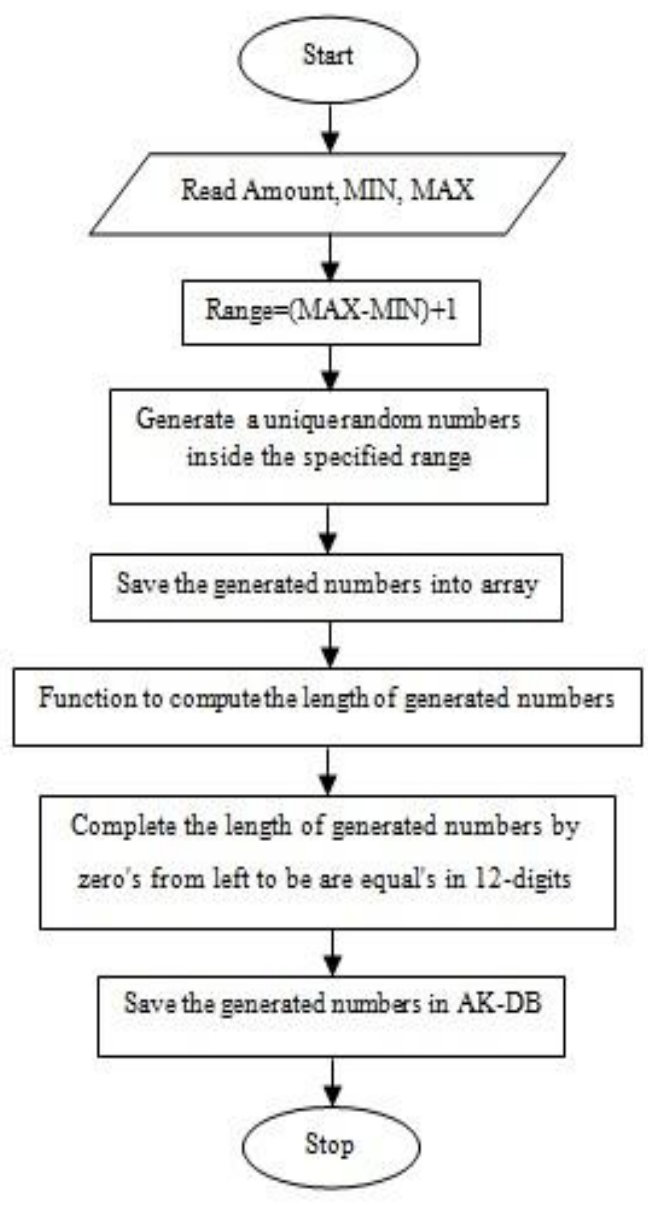

Fig 8: Flowchart for generating AK1and AK2

5. Generate a uniqe random numbers inside the specfied range, about storing the genrated numbers into array to chek if the number exist in the array or not befor storing in the array at each generation step.

6. Store the generated numbers into array

7. Function to compute the length of generated numbers.

8. Complete the length of generated numbers by zero's from left to be are equal's in length's, 12-digits.

\subsubsection{The proposed multi modal algorithm for face recognition:}

In this section, we present our proposed algorithm see fig. 9, for fusing the three appearance-based (or statistical PCA, LDA and SVM) approaches previously described. fig. 1 gives an overview of the proposed method, using database of template (DBT) in every space. for face detection first, an algorithm combining PCV and SVM is used, it is a coarse-tofine process to detect face region. The processing consists of three steps:

- Step_1: face potential is selected using histogram distribution feature. Face and non-face area have different histogram distribution. The histogram of face areas has Gaussian-like distribution but nonface area histogram has irregular distribution.

- Step_2: PCA is used to decrease the dimension of face feature space. At this step, 1000 sample images of size $25 \times 25$ are trained

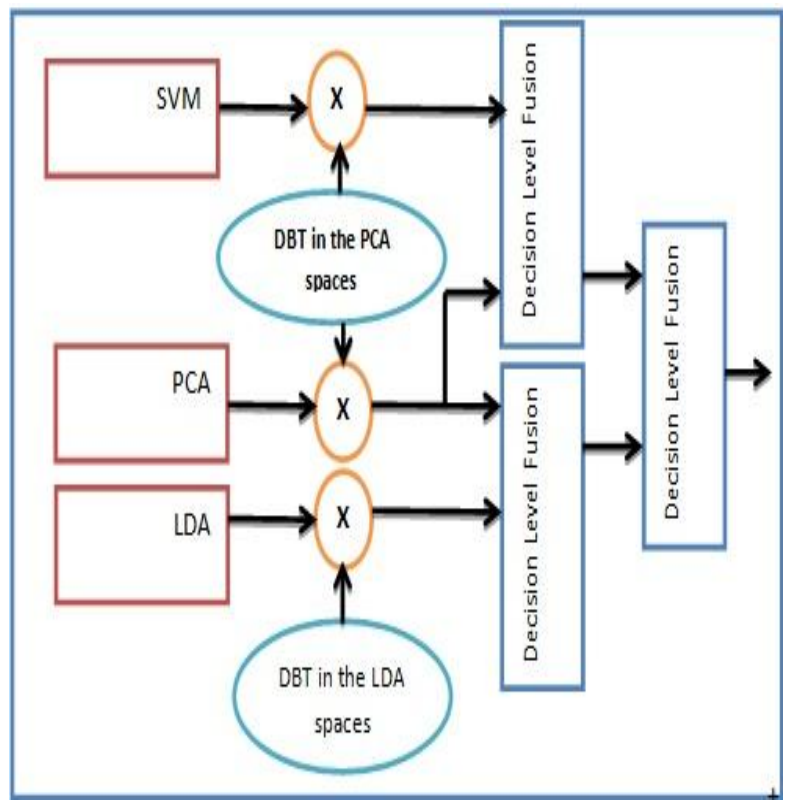

Fig 9: The proposed multi modal algorithm for face Recognition 
- Step_3: SVM is used as classifier to verify face candidate. It is trained by face and non-face samples which are represented by PCA.

Second combining PCA and LDA, where the distance vectors $\mathrm{d}^{\mathrm{PCA}}$ and $\mathrm{d}^{\mathrm{LDA}}$ are defined as $\left(\mathrm{d}_{1}^{\mathrm{PCA}}, \mathrm{d}_{2}^{\mathrm{PCA}}, \ldots, \mathrm{d}_{\mathrm{n}}^{\mathrm{PCA}}\right)$ and $\left(d_{1}^{L D A}, d_{2}^{L D A}, \ldots, d_{n}^{L D A}\right)$ respectively. The result of the decision level fusion of such distance vectors is a novel combined distance vector, which must contain both PCA and LDA information. To combined distance vector as the mean vector. Final step we take the mean of both result from first and second step.

\subsection{Our Model Methodology}

The consumer begins to enter the website if the consumer already registered then the consumer login. However, if the consumer is a new (not register before), then starts by completing the registration form that includes nationality, the social number, mobile number, date of birth address and its image to be uploaded from a file or webcam. Then the national number and mobile number are encrypted so that data can be sent to the TTP as shown in fig. 10.

TTP receives the data fig. 10, and decrypt the user image and mobile number, and then verifying from the data by reference to a governmental body (e.g. civil affairs). If data are not matching, a message will be sent to the consumer mobile number, informing that the data is invalid and try another one. However, if the data is valid, it will be saved in a database peculiar to registration form named App-Fr-DB file, and then given an AK which is taken from AK-DB file that have been generated from Proposed Algorithm as in fig. 7. TTP saves its data in App-Fr-DB file where the image is saved in image-DB file by its AK number. A message will be sent to the consumer mobile phone with AK number to be used in verification of the consumer when making a sale or purchase.

\section{PROPOSED WEB ARCHITECTURE AND SYSTEM IMPLEMENTATION (FACE AUTHENTICATION)}

\subsection{Proposed Web Architecture [20]}

Our proposed web architecture see fig.10, all its components were explained in section 4.2.

\subsection{System Implementation of Face Authentication}

The layout contains two windows, the first window (the registration stage) is shown in fig.11, left section is used for placing webcam window and the generated $\mathrm{AK}$, right section the detected face that will be saved by $\mathrm{AK}$, the second window(the verification stage) fig.12, after verifying from both the $\mathrm{AK}$ and the detected face show the image from DBimage and its AK.

1- The registration Stage:

- Fill the application form see fig. 10.

- Part of the data must be coded before submit (Mobile number and the uploaded user image), and decoded after arriving to the TTP.
- Verify from the input data about the TTP.

- If the input data were verified then generate a user AK.

- Detect the user face (face detection).

- Save the detected user face in database (DBimage) by its user AK, see fig. 11-12.

- Send SMS to the user mobile by its AK.

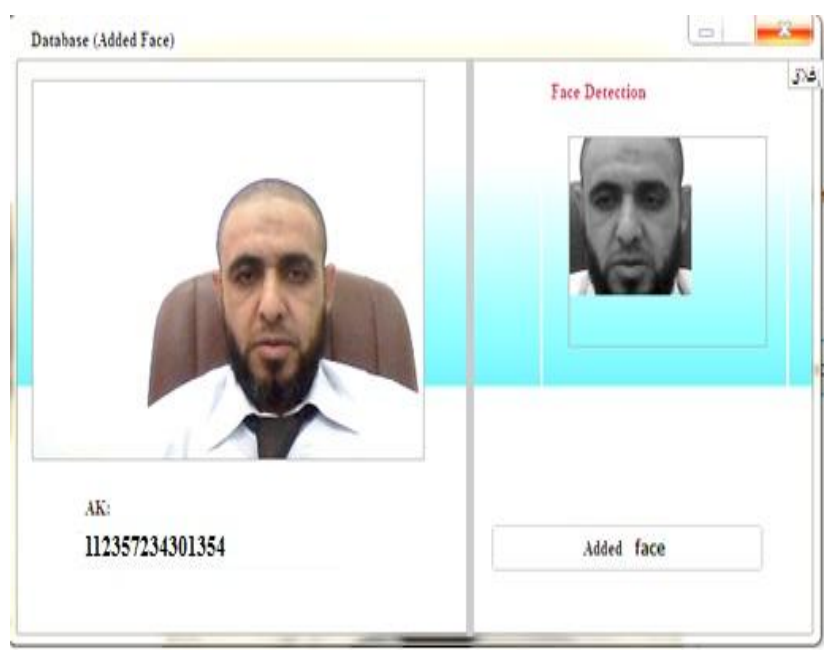

Fig 11: The registration Stage

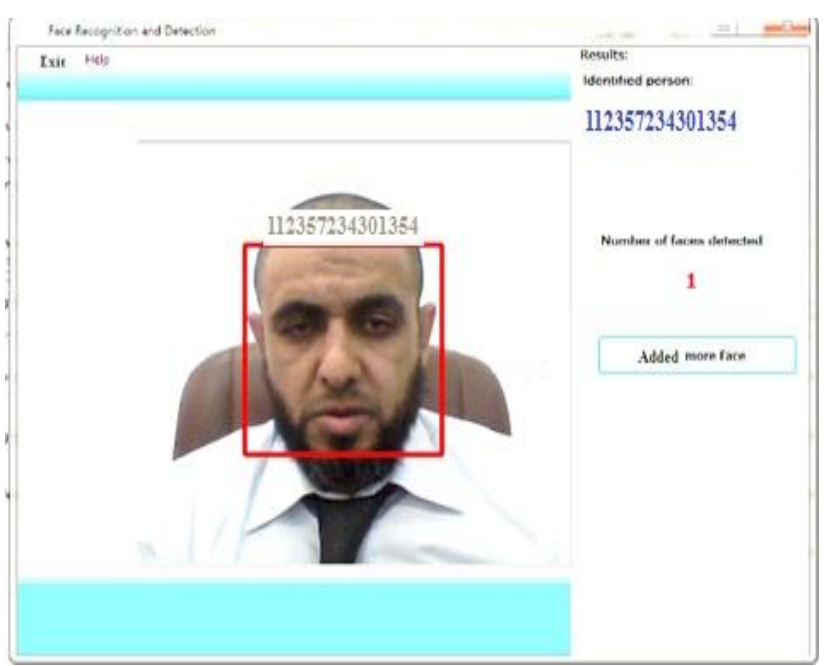

Fig 12: The verification Stage

2-The login stage:

-Enter the user AK.

-If the entered AK true then upload a user image saved in the DBimage by this AK.

-If the face detected from the webcam user image is the same as the face corresponding to AK stored in DBimage(image verification) then welcome by you (you can access the website).

-If one of the entered data are false, then you cannot access the website and he can try for three times before blocked. 


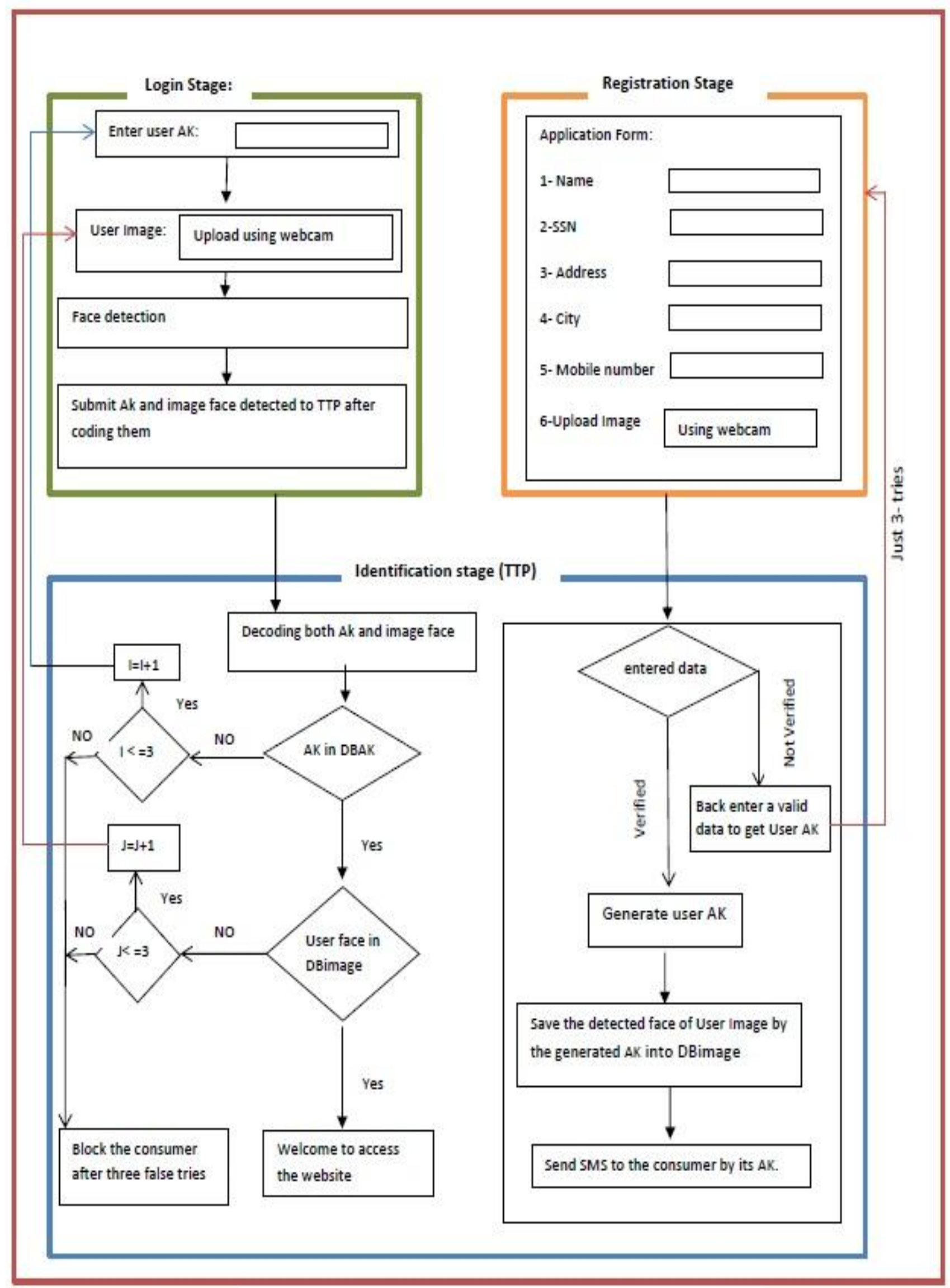

Fig. 10: The Proposed Website Architecture 


\section{CONCLUSION}

This paper aims to increasing the trust in e-commerce about reviewing most of the recent studies (models and website) worked on increasing the trust between consumers (C2C), and make a comparative study for knowing the features and limitations for these websites. Reviewing most of the recent studies on face recognition, a number of algorithms were studied. We modified a previous proposed algorithm for generating AK to be applicable in KSA. Proposing and implementing a multi-modal algorithm of face recognition based on individual algorithms, support vector machine (SVM), principal component analysis (PCA), linear discriminant and analysis (LDA), which more accurate.

Building a system from three stages, first stage: the stage of registration for the first time and where the consumer fills out the application form and coding a part of data (user mobile number and user image) before submit online second stage: The login stages the consumer entering a user $\mathrm{AK}$, and uploads a user image using webcam to $\log$ on to the website. Third stage (verification):in both stages one and two before verification the system decoding the received data, if TTP verify from the data in the first stage, generates a user AK for the user and send it to him, and in the second case, if TTP verify from the login data (authenticity of data) then the consumer can access the operations on the website.

\section{FUTURE WORK}

In future work we will work to evaluate our proposed website architecture, increasing the accuracy of face recognition algorithm and cooperate with a government in building a trusted pay system.

\section{ACKNOWLEDGMENTS:}

This paper is based upon work supported by the Engineering and Applied Sciences, Research Center, Scientific Research Deanship, Majmaah University under Award No. 2-1432.

The author acknowledge the help in conducting various project tasks from Dr. Tawfik Al Kanhal, The Director of the Engineering and Applied Sciences, Research Center.

\section{REFERENCES}

[1] Yaobin Lu, Ling Zhao and Bin Wang," From virtual community members to $\mathrm{C} 2 \mathrm{C}$ e-commerce buyers: Trust in virtual communities and its effect on consumers' purchase intension" Electronic Commerce Research and Applications Volume 9, Issue 4, July-August 2010, Pages 346-360.

[2] Ding, Chunhui Piao, Jing An and Meiqi Fang, "Study on Credit Evaluation Model and Algorithm for C2C ECommerce" 2007 IEEE International Conference on eBusiness Engineering.

[3] Nanchang, "An Approach to Integrating the User Credit Values on Multiple E-Commerce Websites" 2009 Second International Symposium on Electronic Commerce and Security

[4] Jonathan Traupman and Robert Wilensky, "EM-trust: A Robust Reputation Algorithm for Peer-to-Peer Marketplaces", Computer Science Division (EECS) University of California Berkeley, California, July 2005.

[5] Mohammad Usman Ahmed, www.cs.mcgill.ca / mahmed26/eBay_Architecture_Study.pdf.
[6] Dehua HE, Yaobin LU, ZHOU Deyi , Empirical Study of Consumers' Purchase Intentions in C2C Electronic Commerce, ISSN 1007-0214 05/26 pp287-292 Volume 13, Number 3, June 2008.

[7] David S. Marshak, eBay Creates Technology Architecture for the Future , Patricia Seybold Group 2003,http://www.softwaresecretweapons.com/jspwiki/res ources/presentations/Sun_eBay6-2_forWeb.pdf.

[8] www.mudah.my/about/index.htm?ca=9_s.Brown.

[9] http://www.haraj.com.sa/

[10] http://witkey.taskcn.com

[11] Yaobin Lu, Ling Zhao, Bin Wang, "From virtual community members to $\mathrm{C} 2 \mathrm{C}$ e-commerce buyers: Trust in virtual communities and its effect on consumers' purchase intention" Electronic Commerce Research and Applications 9 (2010) 346-360

[12] David S. Marshak, eBay Creates Technology Architecture for the Future , Patricia Seybold Group 2003, http://www.softwaresecretweapons.com/ jspwiki/resources/presentations/Sun_eBay62_forWeb.pdf

[13] http://pages.ebay.com/help/account/questions/aboutebay.html

[14] A. Martinez, A. Kak, "PCA versus LDA", IEEE Transactions on Pattern Analysis and Machine Intelligence, vol. 23, no. 2, pp. 228-233, 2001

[15] A. Hossein Sahoolizadeh, B. Zargham Heidari, and C. Hamid Dehghani, "A New Face Recognition Method using PCA, LDA and Neural Network", International Journal of Electrical and Electronics Engineering 2:8 2008.

[16] A. Hossein Sahoolizadeh, B. Zargham Heidari, and C. Hamid Dehghani, "A New Face Recognition Metusing PCA, LDA and Neural Network", International Journal of Electrical and Electronics Engineering 2:8 2008.

[17] S.Sharavanan , M.Azath, "LDA BASED FACE RECOGNITION BY USING HIDDEN MARKOV MODEL IN CURRENT TRENDS", International Journal of Engineering and Technology Vol.1(2), 2009, $7-85$.

[18] Meina Kan, ShiguangShan , YuSuc, DongXu b, XilinChen, "Adaptive discriminant learning for face recognition", Pattern Recognition, Volume 46, Issue 9, September 2013, Pages 2497-2509

[19] Sharfi M. Abbass, Othman Bin Ibrahim, M. S. Farag "Building a Trust Model for Generating and Validating Assurance Keys between Consumers in E-Commerce" International Journal of Computer Applications (0975 8887)Vol. 57- No.1, November 2012.

[20] Sharfi M. Abbass, Othman Bin Ibrahim, "A model for trust-building in E-commerce from Consumer to Consumer Perspective in KSA" International Journal eEducation, e-Business, e-Management and E-learning Vol. 1, No. 3, Agust2011. 